\title{
A New Look at Generalized Means
}

\author{
Sarah M. Tooth, John A. Dobelman \\ Department of Statistics, Rice University, Houston, TX, USA \\ Email: dobelman@stat.rice.edu
}

Received 28 December 2015; accepted 21 March 2016; published 24 March 2016

Copyright (C) 2016 by authors and Scientific Research Publishing Inc.

This work is licensed under the Creative Commons Attribution International License (CC BY).

http://creativecommons.org/licenses/by/4.0/

(c) $\underset{\mathrm{EY}}{\mathrm{i}}$ Open Access

\begin{abstract}
Since antiquity, the relationships between 2-tuples and their Pythagorean means have been represented in geometric forms. In this paper, we extend the practice to generalized power means through new representations, and also to 3 -tuples. These geometric forms give rise to new algebraic expressions for summary statistics of 2- and 3-tuples.
\end{abstract}

\section{Keywords}

\section{Geometry, Power Means, Pythagorean Means, Outliers}

\section{Introduction}

The study of means dates to antiquity. Pappus of Alexandria, who lived at the end of the third century AD, wrote on the relationships between the "three means" and gave reference to the work of previous geometers [1]. These three means: the arithmetic, geometric, and harmonic, along with a fourth, called the quadratic or root mean squared, represent the most commonly used means even today: the geometric mean finds use in finance and the study of populations, and also in conversion of aspect ratios for film processing; the harmonic mean is used in physics and economics for the treatment of rates and ratios; the quadratic mean is at the heart of ordinary least squares regression, which is used in many diverse fields; and it hardly needs to be said that the arithmetic mean has widespread use as the most used measure of central tendency.

Because of their practical importance, we consider these four means as special named cases of what [2] called “ordinary means" but which are more generally known as power means or generalized means. In this paper, we will modify the notation used by [2] and [3] and define the power means such that for the $N$-tuple $x$ with $x_{i}>0 \forall i$ the $p$-th power mean of $x$ is

$$
M_{p}(x)=\left\{\begin{array}{ll}
\left(\frac{1}{N} \sum_{1}^{N} x_{i}^{p}\right)^{1 / p} & p \in \mathbb{R} \\
\left(\prod_{i}^{N} x_{i}\right)^{1 / N} & p \rightarrow 0 \\
\max x & p \rightarrow \infty \\
\min x & p \rightarrow-\infty
\end{array} .\right.
$$


We find this definition convenient to work with, although we note that the cases given for $p=0, \pm \infty$ represent the limits as the general case approaches those values, as shown by [2]. We also note that, for $p \geq 1$, the power mean is closely related to the $p$-norm, which differs only by a factor of $N^{p}$. The special named cases of the power mean are: arithmetic mean, $M_{p=1}$; geometric mean, $M_{p \rightarrow 0}$; and the harmonic mean, $M_{p=-1}$, which together make the Pythagorean means; and quadratic mean, $M_{p=2}$.

Power means may be regarded as statistics which give more weight to large values of $x_{i}$ when $p$ is large and positive, and more weight to small values of $x_{i}$ when $p$ is large and negative. Because of this property, they can be used comparably with percentiles, but with two important distinctions: the power mean function is continuous and differentiable; and unlike percentiles, power means are not robust to outliers. It is this second property which we consider the most important for its use in the treatment of datasets where outliers are of high importance.

In this paper, we explore geometric representations of power means as a method of investigating the properties of power means. We first describe past geometric representations of the means of 2-tuples, and then introduce our alternative method. Second, we describe how our method can be expanded to represent 3-tuples.

\section{Geometric Representations}

Reference [1] describes how Pappus of Alexandria defines the arithmetic, geometric, and harmonic means according to a series of ratios in his Collection, Book III. Pappus then uses a geometric representation to demonstrate how, if two means are known, the third can always be found. The first geometric representation also comes from Pappus. From [1]:

"Pappus first gives a construction by which another geometer ( $\alpha \lambda \lambda o \varsigma \tau \iota \varsigma$ ) [lit. the other] claimed to have solved this problem, but he does not seem to have understood it, and returns to the same problem later".

It is not Pappus's solution, but that of the unnamed geometer, with the addition of the quadratic mean by [4], which has become the common form. It is shown in Figure 1. This form is limited to represent the means of a 2-tuple, herein $x$. If we set $A B=\max x_{i}$ and $B C=\min x_{i}$, then $M_{1}=F O, M_{0}=B D, M_{-1}=D E$ and $M_{2}=B F$.

We firstly propose a simple extension of the traditional representation in Figure 1 which allows the representation of any $p \in \mathbb{R}$. We do this by removing the lines assigned to particular cases and introducing a single variable length which can equal any value of $M_{p}$. This modified form is shown in Figure 2. Here again, $A B=$ $\max x_{i}$ and $B C=\min x_{i}$, but in this new representation $B D(\theta=g(p))=M_{p}$, where $g(p)$ is a sigmoid function with domain $(-\infty, \infty)$ and range $[\min (x), \max (x)]$.

Setting $A B=a ; B C=b$, and solving Figure 2 for $M_{p}$ gives:

$$
M_{p}=B D(\theta)=\frac{(b-a) \cos \theta+\sqrt{4 a b+(b-a)^{2} \cos ^{2} \theta}}{2} .
$$

While it may be debated whether Equation (2) is simpler to work with for general purposes than Equation (1), it has the clear advantage of being invertible. While we must solve Equation (1) for $p$ numerically, solving Equation (2) for $\theta$ gives the closed form:

$$
\theta=\arccos \frac{M_{p}^{2}-a b}{(b-a) M_{p}}
$$

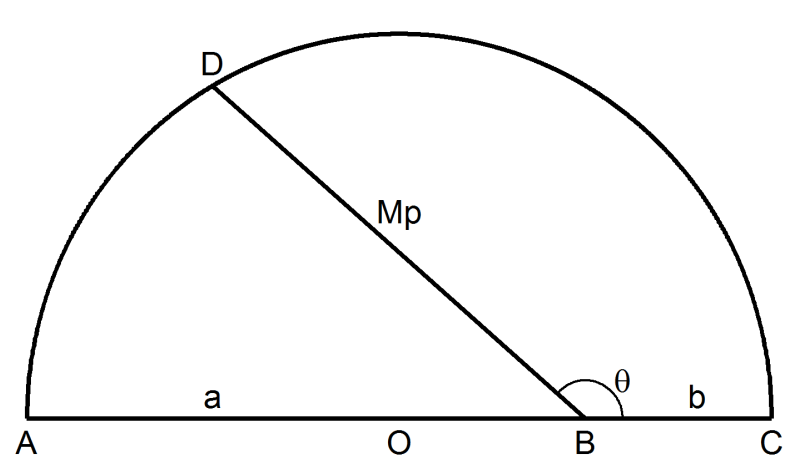

Figure 1. Traditional geometric representation of means. 
A sampling of values of $p, M_{p}$ and $\theta$ when $a=3$ and $b=1$ are shown in Table 1. For all $a$ and $b$, there are three fixed points in the mapping between $M_{p}$ and $B D(\theta)$ which are $M_{-\infty}=B D(0), M_{0}=B D\left(\frac{\pi}{2}\right)$ and $M_{\infty}=B D(\pi)$.

To construct a geometric representation of the power means of a 3-tuple $x$ as a natural extension of our $N=2$ representation, we establish a number of desirable criteria. An $N=3$ representation should have: a) an arrangement of line segments with lengths equal to each element $x$ in the series; $b$ ) a curved path, on which any position can be described using only an angle $\theta$; c) a line segment $M$ with a length that is a function of $\theta$, that is, $|M|=f(\theta)$ where $f$ is monotone increasing; and d) a geometry such that $0 \leq \theta \leq \pi, f(0)=\min (x)$ and $f(\pi)=\max (x)$. The resulting representation is shown in Figure 3 .

In Figure 3, the dimensions are assigned as follows: $A B=\max x, B C=\min x, B D=M$. While it will be intuitive to assign $A C=$ med $x$ and thus form a triangle of the elements of $n$, the simple example $x=1,2,5$ illustrates why this is not possible. Because $\max x>\min x+\operatorname{med} x$, we cannot form a triangle with these three lengths.

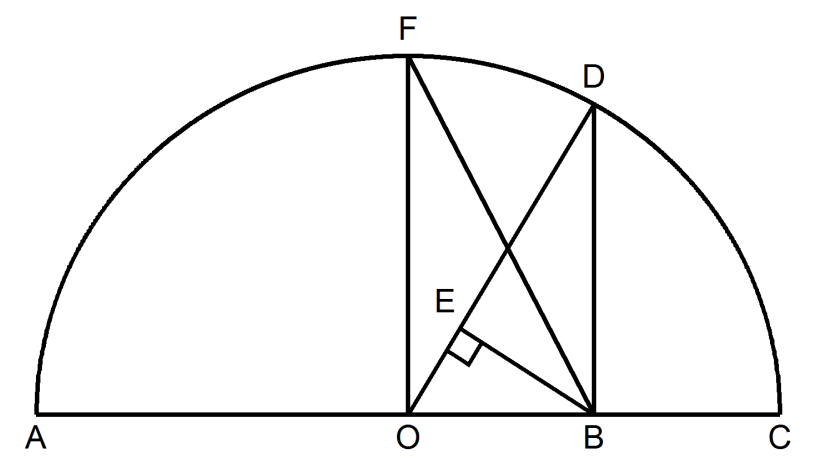

Figure 2. Modified geometric representation of power means.

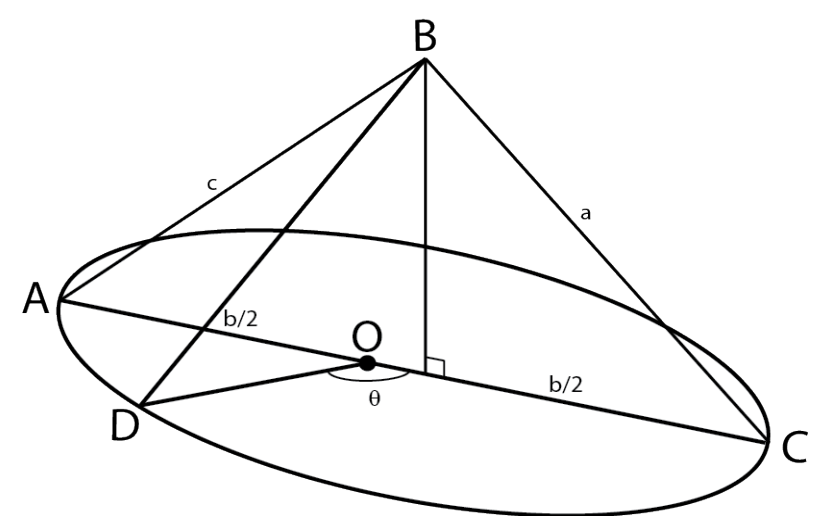

Figure 3. Representation for power means of 3-tuples.

Table 1. Sample values for $a=3 ; b=1$.

\begin{tabular}{ccc} 
& Example Values & \\
$p$ & $M_{p}$ & $\theta$ \\
\hline$-\infty$ & 1.000 & 0.000 \\
-1 & 1.500 & 1.318 \\
0 & 1.732 & $\pi / 2$ \\
1 & 2.000 & 1.823 \\
2 & 2.236 & 2.034 \\
$\infty$ & 3.000 & $\pi$ \\
\hline
\end{tabular}


However, solving the system in Figure 3 gives Equation (4), which shows that our power mean, as represented by $B D$, is independent of $A C$ (i.e. independent of med $x$ ).

$$
M_{p}=B D(\theta)=\sqrt{a^{2} \cos ^{2}(\theta / 2)+c^{2} \sin ^{2}(\theta / 2)}
$$

A sampling of values of $p, M_{p}$ and $\theta$ when $a=1$ and $c=3$ is shown in Table 2. As with the 2-tuple case, there are three fixed points in the mapping between $M_{p}$ and $B D(\theta)$ for all $a$ and $c . M_{-\infty}=B D(0)$ and $M_{\infty}=B D(\pi)$ are consistent with the 2-tuple case, but rather than a fixed point at $M_{0}$, the 3-tuple has a fixed point at $M_{2}=B D\left(\frac{\pi}{2}\right)$.

One consequence of this relationship is that, if we set $A C=A B-B C$, then our $N=3$ system in Figure 3 becomes Figure 4, which has a greater resemblance to the $N=2$ representation from [4], shown in Figure 5, than our own $N=2$ representation in Figure 2. Note that unlike previous $N=2$ representations where the semicircle has a diameter of $\max x+\min x$, these figures have semi-circles with diameter $\max x-\min x$.

$$
\begin{aligned}
& \frac{\mathrm{d} M_{p}}{\mathrm{~d} \theta}=\frac{\left(c^{2}-a^{2}\right) \sin \theta}{2 \sqrt{2} \sqrt{a^{2}+c^{2}+\left(a^{2}-c^{2}\right) \cos \theta}} \\
& \theta=\arccos \frac{2 M_{p}^{2}-a^{2}-c^{2}}{a^{2}-c^{2}}
\end{aligned}
$$

Like the traditional form in Equation (1), Equation (4) is differentiable (Equation (5a)). It is also invertible, (Equation (5b)) a property which the traditional form lacks.

Finally, a comparison of Figure 2 and Figure 3 suggests characteristics of a similar representation of means for $N$-tuples for $N \geq 4$. In both figures, $N$ segments are arranged in $N-1$ dimensions, and the mean $M_{p}$ can be represented as the distance between one vertex and a semi-circle which lies in an orthogonal plane and connects two other vertices. As a result, the entire system can be represented as a semi-circle and a triangle which lie in two orthogonal planes, where the triangle is a projection from $N-1$ to 2 dimensions. It follows,

Table 2. Sample values for $a=1 ; c=3$.

\begin{tabular}{ccc}
\hline & Example Values & \\
\hline$p$ & $M_{p}$ & $\theta$ \\
\hline$-\infty$ & 1.000 & 0.000 \\
-1 & 1.500 & 0.813 \\
0 & 1.732 & 1.047 \\
1 & 2.000 & 1.318 \\
2 & 2.236 & $\pi / 2$ \\
$\infty$ & 3.000 & $\pi$ \\
\hline
\end{tabular}

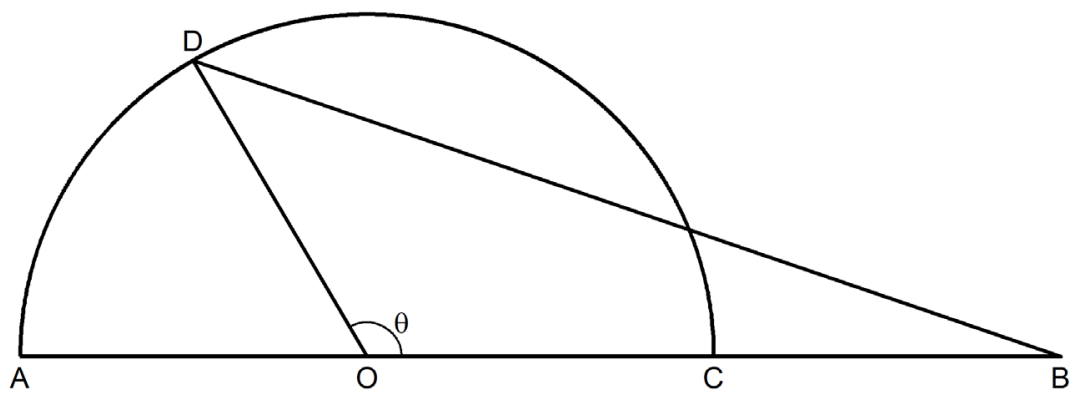

Figure 4. Projection of modified representation for 3-tuples. 


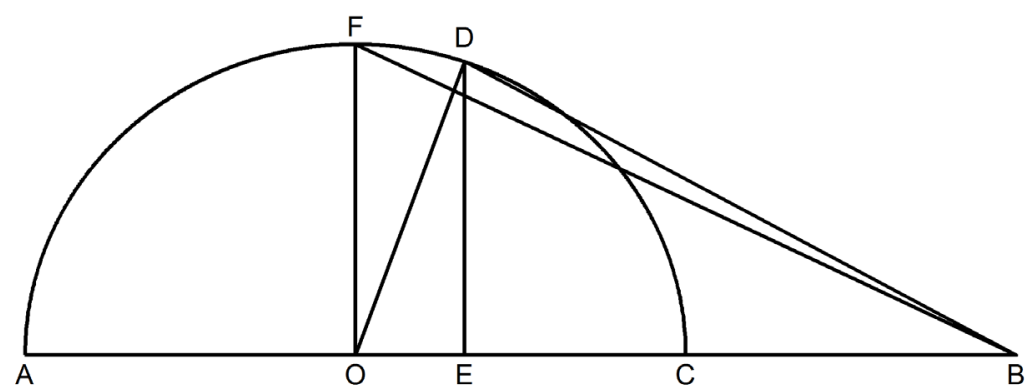

Figure 5. Modified representation for 2-tuples by Ercolano (1972).

then, that provided both $\max x_{i}$ and $\min x_{i}$ are unchanged, each additional $x_{i}$ will have progressively less influence on the overall shape of the function. This same behavior is seen in the algebraic form given in Equation (1), implying that the geometric representation has similar limiting properties to the algebraic.

\section{Acknowledgements}

The authors would like to thank Rice University Professor Frank Jones, as well as William Longley and Reid Atcheson, for helpful discussions.

\section{References}

[1] Heath, T. (1921) Greek Mathematics. Vol. 2, Oxford University Press, Cambridge.

[2] Hardy, G., Littlewood, J. and Pólya, G. (1934) Inequalities. Cambridge University Press, Cambridge.

[3] Bullen, P. (2003) Handbook of Means and Their Inequalities. Springer, Netherlands. http://dx.doi.org/10.1007/978-94-017-0399-4

[4] Ercolano, J. (1972) Geometric Interpretations of some Classical Inequalities. Mathematics Magazine, 45, 226. 